\title{
SignalP Algorithm
}

National Cancer Institute

\section{Source}

National Cancer Institute. SignalP Algorithm. NCI Thesaurus. Code C49043.

An algorithm that predicts the presence and location of signal peptide cleavage sites in amino acid sequences. It incorporates a prediction of cleavage sites and a signal peptide/non-signal peptide prediction based on a combination of several artificial neural networks and hidden Markov models. 